\title{
Rare Case of Cecal Signet Ring Cell Carcinoma with Distal Intramural Spread
}

\author{
KOHEI ONO ${ }^{1}$, MASAHIKO MURAKAMI ${ }^{1}$, MAKOTO WATANABE ${ }^{1}$, AKIRA FUJIMORI $^{1}$, \\ KOJI OTSUKA ${ }^{1}$, TAKESHI AOKI ${ }^{1}$, TOSHIKO YAMOCHI-ONIZUKA ${ }^{2}$ and MASAFUMI TAKIMOTO ${ }^{2}$ \\ ${ }^{1}$ Department of Gastroenterological \& General Surgery, School of Medicine, Showa University, Tokyo, Japan; \\ ${ }^{2}$ Department of Pathology, School of Medicine, Showa University, Tokyo, Japan
}

\begin{abstract}
In this paper, we report a rare case of cecal Signet ring cell carcinoma (SRCC) with Distal intramural spread (DIS) along with a review of the literature. A 71-yearold woman suffering from vomiting, abdominal pain, and abdominal distension was admitted to a hospital and was suspected to have ileus. She was transferred to our hospital and diagnosed with cecal cancer with intestinal obstruction. Laparotomy was performed, after which she was diagnosed with cecal SRCC by histopathological examination. A submucosal lesion was located $55 \mathrm{~mm}$ from the distal side of the main tumor. This lesion was also diagnosed as SRCC. It was not exposed to the epithelium or the serous membrane. The submucosal tumor was diagnosed as DIS of cecal SRCC. After the operation, she underwent chemotherapy with FOLFIRI+Cet (5-fluorouracil, leucovorin, and irinotecan plus cetuximab). At a follow-up examination nine months after surgery, she was found to be doing well.
\end{abstract}

The existence of microscopic tumor implants in the intestinal wall, distal to the inferior edge of a macroscopic tumor (either continuous or discontinuous with the tumor), is called distal intramural spread (DIS) (1). The rate of DIS in rectal cancer is reported as $14.7 \%$ (2) to $83.6 \%$ (3). On the contrary, DIS has not been reported in colonic cancer and is considered rare. DIS is classified as continuous or discontinuous lesions. Discontinuous lesions spread from the distal side of the main tumor through the normal epithelium. These lesions are called skip lesions (4). Skip lesions are

This article is freely accessible online.

Correspondence to: Kohei Ono, Department of Gastroenterological \& General Surgery, School of Medicine, Showa University, 1-5-8, Hatanodai, Shinagawa-ku, Tokyo, 1428666, Japan. Tel: +81 337848541, e-mail: co-hey.o@med.showa-u.ac.jp

Key Words: Cecum, signet ring cell carcinoma, distal intramural spread. thought to invade the submucosal or muscular layers through the lymphatic vessels $(5,6)$.

More than $90 \%$ of colorectal carcinomas are adenocarcinomas originating from epithelial cells of the colorectal mucosa (7). According to a previous report (8), $2.63 \%$ of patients with colonic cancer had Signet ring cell carcinomas (SRCC). In this study we report a rare case of cecal SRCC with DIS diagnosed pathologically.

\section{Case Report}

A 71-year-old woman with hypertension and dyslipidemia was referred to another hospital with vomiting, abdominal pain, and abdominal distension. She was suspected of having ileus and was transferred to our hospital. Contrast enema showed a cecal mass. There was no flow of contrast medium into the ileum. Colonoscopy showed complete stenosis of the ascending colon. Pathological examination showed SRCC. Upper gastrointestinal endoscopy did not indicate any neoplastic lesion. Computed tomography (CT) of the abdomen showed a thickening of the cecal wall with fluid accumulation in the distal ileum. Swelling of lymph nodes around the cecum, ascites, and peritoneal metastasis were observed (Figure 1). The level of carcinoembryonic antigen (CEA) was $6.8 \mathrm{ng} / \mathrm{ml}$. Other laboratory results were normal. She was clinically diagnosed with cecal cancer, which caused ileus and carcinomatous peritonitis. Surgery was performed by iliocecal resection with lymphadenectomy. Pathological examination of the resected specimen showed SRCC invading all layers of the cecal wall. There was massive invasion of the lymphatic vessels and venous vessels (Figure 2) and metastasis to the lymph nodes.

A submucosal nodule (diameter, $10 \mathrm{~mm}$ ) was located $55 \mathrm{~mm}$ from the distal side of the main tumor through the normal epithelium (Figure 3). This lesion was also diagnosed as SRCC. It was located in the muscularis propria to subserosa. Because no carcinoma cells were exposed to the epithelium or serosa, we diagnosed the lesion as DIS of cecal SRCC (Figure 4). The final pathological stage was 

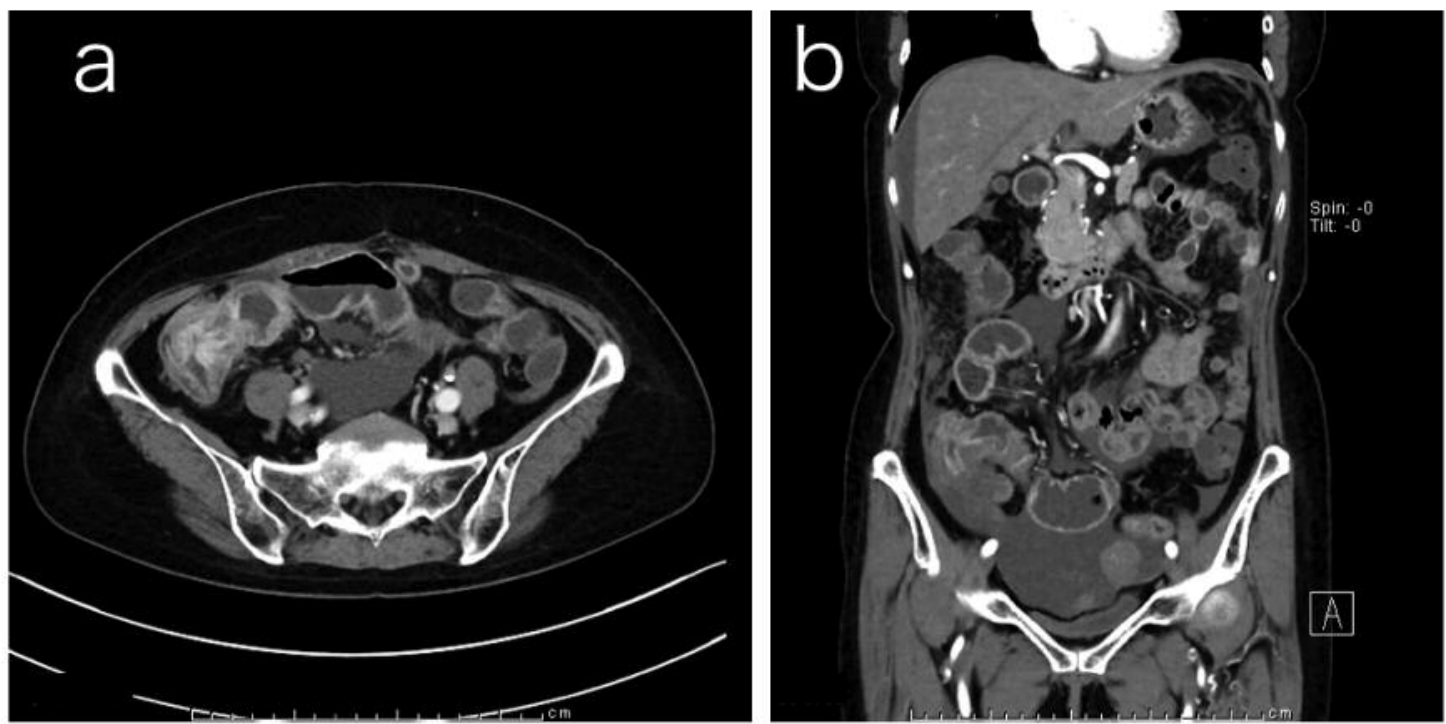

Figure 1. The initial abdominal CT findings. CT of abdomen showed a thickening of the cecal wall with distal ileal fluid accumulation. There was also swelling of the lymph nodes and ascites. a: Axial section; $b$ : coronal section.
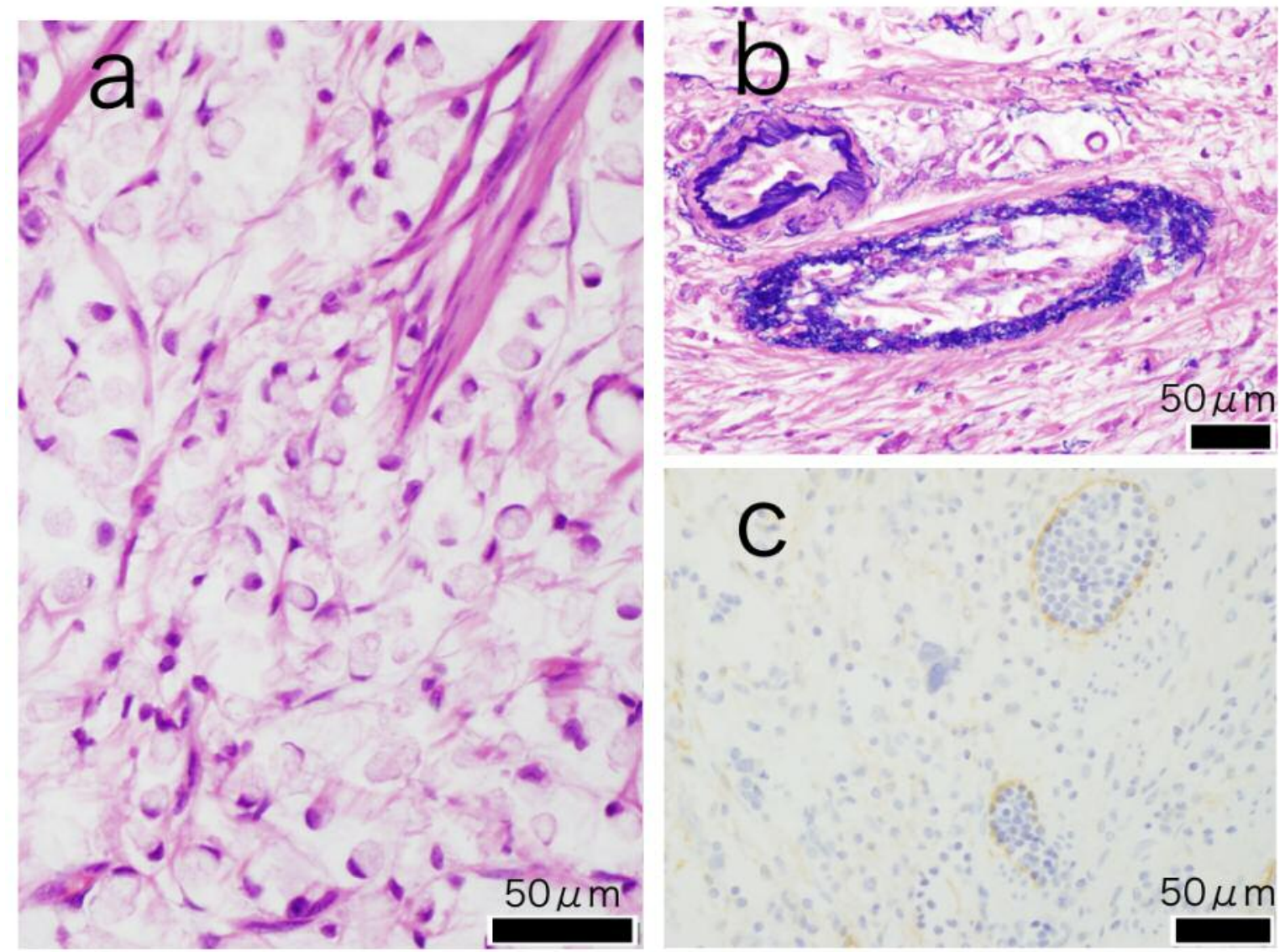

Figure 2. The histopathological findings of the main tumor. a: SRCC (hematoxylin-eosin) (x400). b: Massive invasion of carcinoma cells in lymphatic vessels (D2-40) (×400). c: Massive invasion of carcinoma cells in veins (Victoria blue) $(\times 400)$. 

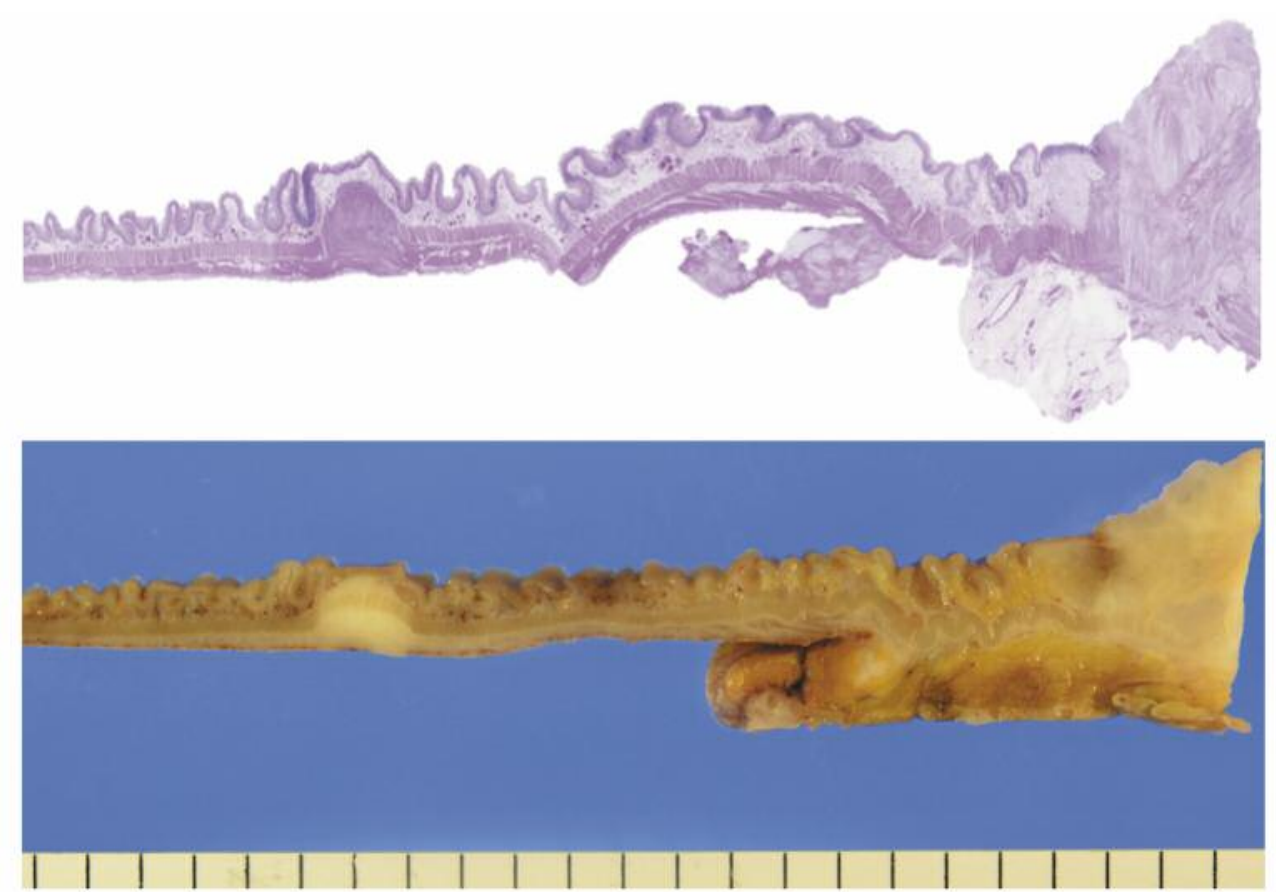

Figure 3. Macroscopic image of the cecum and ascending colon. Nodular lesion was located $55 \mathrm{~mm}$ from the distal side of the main tumor.

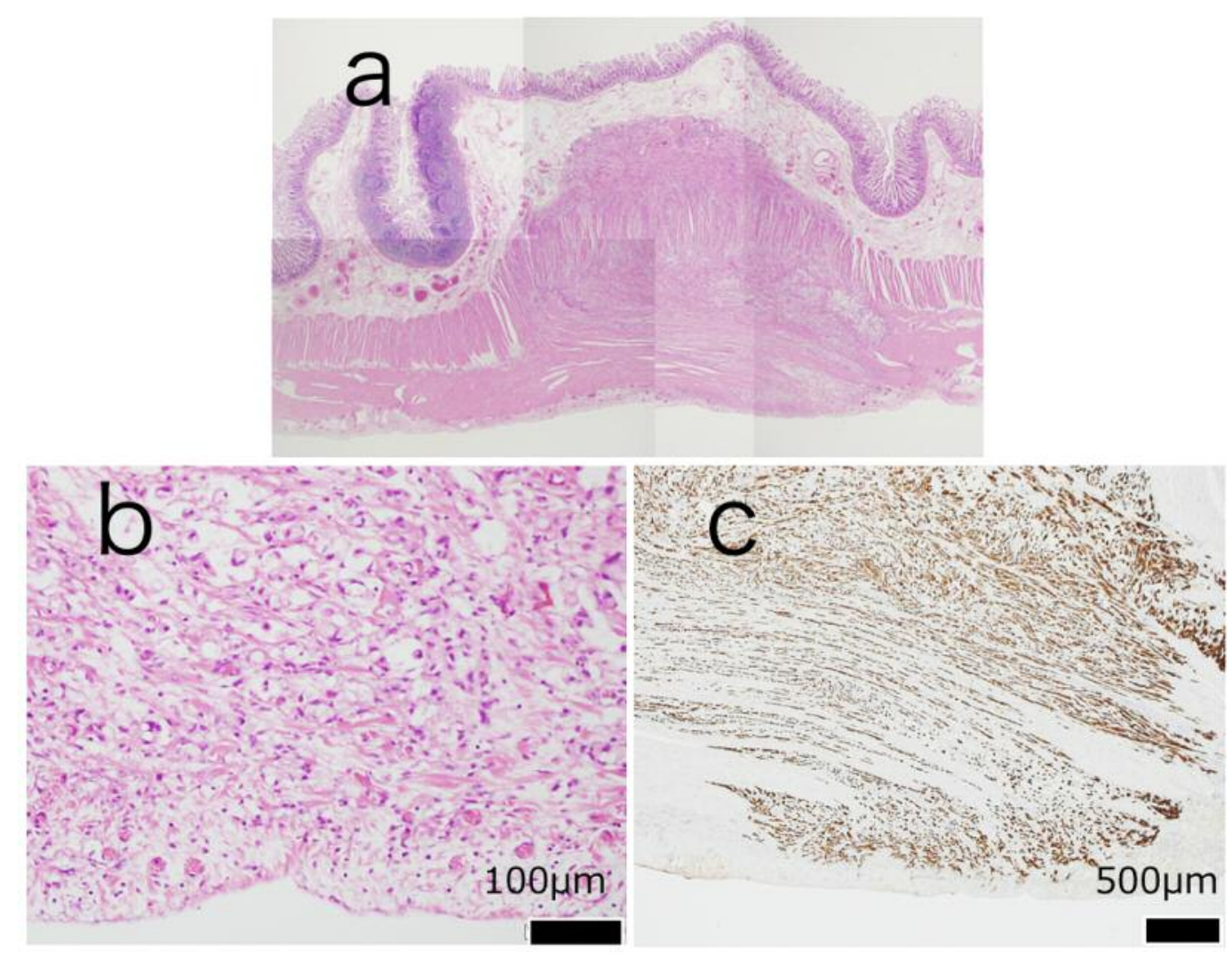

Figure 4. Histopathological findings of the DIS. a: Nodular lesion was located in the muscularis propria to subserosa (hematoxylin-eosin) ( $\times 40)$. b: Signet ring cell carcinoma (hematoxylin-eosin) (x400). c: No extraserosal invasion of carcinoma cells $(C K$ AE1/AE3) ( $\times 400)$. 
T4aN2bM1b (stage IVB according to the Union for International Cancer Control).

The patient had no postoperative complications and was discharged on postoperative day 10. She underwent chemotherapy with FOLFIRI+Cet (5-fluorouracil, leucovorin, and irinotecan plus cetuximab) as an outpatient. She is now well 9 months after surgery.

\section{Discussion}

There are many reports of DIS in the rectum (1-3, 5, 9-11) but few of DIS in the colon. A search of PubMed from 1990 to 2017 found only one case of DIS of colonic malignant lymphoma with intussusceptions (12). Thus, the present case is the first report of DIS of colonic epithelial neoplasm.

DIS is a type of cancerous metastasis that is regarded not only as a marker to identify the surgical margin during operation (1) but also as an index for the prognosis of rectal cancer (12). It has also been reported to be related to the depth of cancer invasion (5). Furthermore, DIS has a potential for metastasis after radical resection. The diseasefree survival rate of patients with DIS is lower than that of patients without DIS (13). DIS is noticed as a submucosal lesion (9). In a previous report of DIS of rectal cancer, the size of the DIS was less than $1 \mathrm{~cm}$ in $78.4 \%$ of cases, greater than $2 \mathrm{~cm}$ in $6 \%$, and greater than $3 \mathrm{~cm}$ in only $1.6 \%$ (9). It is difficult to detect DIS by colonoscopy or CT on preoperative screening. Ultrasonography has been recommended for detection of DIS (10).

In rectal cancer, lymphatic invasion occurs via the proximal, lateral, and distal routes (11). The proximal flow is associated with the inferior mesenteric lymph nodes, the lateral with the pararectal lymph nodes, and the distal with the inguinal lymph nodes. Tumor cells invade through the lymphatic passages. It is assumed that abundant lymphatic flow is associated with DIS. In our case, the DIS lesion was found $55 \mathrm{~mm}$ from the distal side of the main tumor by macroscopic search. There was massive invasion of SRCC into lymphatic vessels and venous vessels.

We could not detect any dysplastic change in the epithelium of the lesion macroscopically or microscopically. It is difficult to detect a DIS lesion before surgery. When we diagnose advanced colonic cancer, we should always keep the possibility of DIS as a submucosal lesion.

In conclusion, we report a case of cecal SRCC with DIS. To the best of our knowledge, this is the first report of such a case.

\section{References}

1 Ignacio Raffa C, Schmitz L, Fandiño S, Sánchez PJ and Bumaschny E: Distal intramural spread of adenocarcinoma of the rectum after neoadjuvant therapy. Acta Gastroenterol Latinoam 44: 299-304, 2014.

2 Shimada Y, Takii Y, Maruyama S and Ohta T: Intramural and mesorectal distal spread detected by whole-mount sections in the determination of optimal distal resection margin in patients undergoing surgery for rectosigmoid or rectal cancer without preoperative therapy. Dis Colon Rectum 12: 1510-1520, 2011.

3 Pan ZZ, Wan DS, Zhang CQ, Shao JY, Li LR, Chen G, Zhou ZW and Wang FL: Using p53-immunostained large specimens to determine the distal intramural spread margin of rectal cancer. World J Gastroenterol 12: 1626-16269, 2006.

4 Lazorthes F, Voigt JJ and Roques J: Distal intramural spread of carcinoma of the rectum correlated with lymph nodal involvement. Surg Gynecol Obstet 1: 45-48, 1990.

5 Andreola S, Leo E, Belli F, Lavarino C, Bufalino R, Tomasic G, Baldini MT, Valvo F, Navarria P and Lombardi F: Distal intramural spread in adenocarcinoma of the lower third of the rectum treated with total rectal resection and coloanal anastomosis. Dis Colon Rectum 40: 25-29, 1997.

6 Chen W, Shen W, Chen M, Cai G and Liu X: Study on the relationship between lymphatic vessel density and distal intramural spread of rectal cancer. Eur Surg Res 39: 332-329, 2007.

7 Hamilton SR, Bosman FT and Boffetta P: Carcinoma of the colon and rectum. In: WHO Classification of tumours of the digestive system. IARC Press: 134-146, 2010.

8 Fu J, Wu L, Jiang M, Tan Y, Li D, Chen F, Jiang T and Du J: Signet ring cell carcinoma of resectable metastatic colorectal cancer has rare surgical value. J Surg Oncol 114: 1004-1008, 2016.

9 Williams NS, Dixon MF and Johnston D: Reappraisal of 5 centimetre rule of distal excision for carcinoma of the rectum: a study of distal intramural spread and of patients' survival. Br J Surg 70: 150-154, 1983.

10 Yanagi H, Kusunoki M and Shoji Y: Preoperative detection of distal intramural spread of lower rectal carcinoma using transrectal ultrasonography. Dis colon Rectum 11: 1210-1214, 1996.

11 Yu YY, Wang C, Xu D, Shen XG, Ding SQ and Zhou ZG: Mesorectal and lateral node metastasis and micrometastasis in lower rectal cancer. Hepatogastroenterology 58: 745-748, 2011.

12 Takiguchi N, Sarashina H, Saitoh N, Nunomura M, Kouda K, Ozaki K, Nakajima N, Fujihira T and Mikata A: Ileocolic intussusception in adult due to malignant lymphoma in the cecum with intramural metastasis. J Gastroenterol 31: 603-606, 1996.

13 Hayashi N, Ito I, Yanagisawa A, Kato Y, Nakamori S, Imaoka S, Watanabe H, Ogawa M and Nakamura Y: Genetic diagnosis of lymph-node metastasis in colorectal cancer. Lancet 345: 12571259, 1995.

Received April 13, 2017

Revised April 27, 2017

Accepted April 28, 2017 